\title{
Comparative Assessment of Growth and Gene Regulation between 4 Serotypes of Streptococcus pneumoniae in Broth and Cell Culture
}

\author{
Ben Seabolt ${ }^{1}$, Jeff Brady ${ }^{2}$, Janice Speshock ${ }^{*}$ \\ ${ }^{1}$ Department of Biological Sciences, Tarleton State University, Stephenville, TX, USA \\ ${ }^{2}$ Texas A\&M AgriLife Research and Extension Center, Stephenville, TX, USA \\ Email: *speshock@tarleton.edu
}

How to cite this paper: Seabolt, B., Brady, J. and Speshock, J. (2018) Comparative Assessment of Growth and Gene Regulation between 4 Serotypes of Streptococcus pneumoniae in Broth and Cell Culture. Advances in Microbiology, 8, 763-777. https://doi.org/10.4236/aim.2018.810050

Received: May 7, 2018

Accepted: October 5, 2018

Published: October 8, 2018

Copyright (c) 2018 by authors and Scientific Research Publishing Inc. This work is licensed under the Creative Commons Attribution International License (CC BY 4.0).

http://creativecommons.org/licenses/by/4.0/

\begin{abstract}
Streptococcus pneumoniae is a medically important pathogen capable of causing human infections of pneumonia, bacteremia, otitis media, and meningitis. Although there are vaccinations available, infections with $S$. pneumoniae still remains a global problem. $S$. pneumoniae is a highly adaptable bacterial species with numerous serotypes based on capsular polysaccharides. The different serotypes vary in their ability to colonize and causing pathology. Here we compared the regulation of five different virulence factors from four common serotypes of $S$. pneumoniae that vary in their carriage, morbidity, and mortality rates in the human population using two different in vitro methods, broth and cell culture. We determine that there is variation of virulence factor gene regulation within a serotype using two different culture methods, and variation between the serotypes in the same culture condition. The regulation of genes appeared to have a correlation with the ability of the various serotypes to grow in broth culture, adhere to cultured lung cells, and invade the cultured lung cells, as serotypes that shared similar regulation of virulence factors tended to behave similarly in culture. Many studies with $S$. pneumoniae rely on the use of one selected serotype, but since there is a wide variation in the growth and regulatory mechanisms of these bacteria. As demonstrated here, future studies should utilize more strains in models before concluding mechanisms of pathobiology.
\end{abstract}

\section{Keywords}

Pneumococcus, Serotypes, Gene Expression, Regulation 


\section{Introduction}

Streptococcus pneumoniae is a transient, opportunistic pathogen found in the upper respiratory tracts of children and adults. $S$. pneumoniae can result in serious pathology, but is rare in healthy individuals and tends to cause morbidity and mortality in mainly those who are under two years of age, over 60 years of age, or are otherwise immunocompromised [1] [2]. However, there are still nearly 1 million cases of severe pneumococcal disease annually in the United States [3] even with available pneumococcal vaccinations [4]. The global mortality resulting from this bacterial species is substantially higher, especially in those countries with poor access to the pneumococcal vaccines [5].

There are more than 90 serotypes of $S$. pneumoniae classified by the structure of their polysaccharide capsule [6]. Disease severity and carriage rate are highly impacted by serotype, and several serotypes have been more highly implicated in causing majority of the severe pneumococcal diseases such as meningitis and bacteremia [7]. While it is known that host immune responses play a significant role in the severity of pneumococcal disease, it is also hypothesized that the expression of virulence genes in $S$. pneumonia will contribute to colonization and pathology. A gene expression study was performed to assess the variation in expression between four different $S$. penumoniaeserotypes using the first component of the capsule operon ( $c p s A)$, and the virulence factors choline-binding proteins $(c b p A)$, autolysin $(l y t A)$, pneumolysin $(p l y)$, and hemolysin $(s p x B)$. The pneumococcal capsule is made up of a repeating polysaccharide sequence unique to each serotype, yet the arrangement of genes within the capsule loci, designated cps $A-D$, is conserved [8]. CbpA, is a highly-conserved adhesin that allows $S$. pneumoniae to translocate across nasopharyngeal epithelial cells and invade the circulatory system [9]. The pneumococcal autolysin (lytA) hydrolyzes the peptidoglycan cell wall to allow for the release of the pneumolysin [10]. The pneumolysin is a pore-forming toxin expressed by the ply gene in virtually all pneumococcal serotypes. At high concentrations, pneumolysin creates holes in cholesterol-containing cell membranes; at lower, sub-lytic concentrations it has a host of pro-inflammatory effects which aid in bacterial dissemination [11] [12]. SpxBis involved in the reduction of $\mathrm{O}_{2}$ to $\mathrm{H}_{2} \mathrm{O}_{2}$ [13], and decreased activity of $s p \times B$ leads to increased capsule production [14]. S. pneumoniae uses pyruvate oxidase as a way to measure the oxygen level of the immediate environment and regulates the level of capsule production based on the oxygen levels [14].

This study compared the growth and gene expression between two invasive serotypes ( 3 and $9 \mathrm{~V}$ ) to two less pathogenic serotypes ( 5 and $19 \mathrm{~F}$ ) in vitro to compare the expression of the virulence factors described above. A broth culture was used to induce expression, as well as a human lung epithelial cell line (A549) to examine differences in gene expression impacted by the presence of eukaryotic host cells. The differential gene regulation was then compared to the growth phenotypes of the strains in broth culture as well as the ability to adhere or invade A549 cells. 


\section{Materials and Methods}

\subsection{Bacterial Strains}

Strains6303 (serotype 3), 6305 (serotype 5), 700671 (serotype 9 v), and 49619 (serotype 19 F) were obtained from the American Tissue Culture Collection (ATCC; Manassas, VA). The cultures were grown by inoculating Trypticase Soy Broth (TSB; Becton Dickinson, Franklin Lakes, NJ) and incubating at $37^{\circ} \mathrm{C}$ overnight. The cultures were frozen in a $25 \%$ glycerol solution at $-80^{\circ} \mathrm{C}$ until needed.

\subsection{Growth Curves in Broth}

Each serotype (3, 5, $9 \mathrm{v}$, and $19 \mathrm{~F})$ was grown by adding 200 microliters $(\mu \mathrm{L})$ of overnight culture to 10 milliliters (mL) of trypticase soy broth (TSB) and incubated at $37^{\circ} \mathrm{C}$ for 8 hours to compare growth rates of the different serotypes. At every hour the optical density (OD) was measured via spectrophotometry using a Beckman DU530 spectrophotometer (Beckman Coulter, Brea, CA) for each replicate $(n=4)$ at a wavelength of $600 \mathrm{~nm}$.

\subsection{RNA Isolation from Broth Cultures}

Ten $\mathrm{mL}$ of TSB was inoculated with $10 \mu \mathrm{L}$ of culture for each of the four serotypes and was incubated at $37^{\circ} \mathrm{C}$ overnight. The following morning $10 \mathrm{~mL}$ of fresh TSB for each serotype was inoculated with $200 \mu \mathrm{L}$ and was grown at $37^{\circ} \mathrm{C}$ to mid-logarithmic phase ( $\mathrm{OD}=0.250)$. When log phase was reached, the incubation was stopped and the cells were centrifuged at $4000 \times \mathrm{g}$ for 5 minutes to obtain a cell pellet (Eppendorf 5810R, Hamburg, Germany). The supernatant was discarded and the cells were re-suspended in $350 \mu \mathrm{L}$ tri-reagent (Sigma Aldrich, St. Louis, MO). The samples were vortexed for approximately two minutes, and then allowed to sit at room temperature for five minutes. Then the samples were vortexed for two additional minutes which was followed by a one minute centrifugation at $11,500 \times \mathrm{g}$ (Eppendorf 5810R, Hamburg, Germany). The supernatant was transferred to a new tube and $350 \mu \mathrm{L}$ of $100 \%$ ethanol was added. The contents were transferred to an RNA mini spin column (Enzymax LLC, Lexington, KY) and centrifuged for one minute at $1000 \times \mathrm{g}$. The resulting flow-through was discarded and $700 \mu \mathrm{L}$ of high stringency wash buffer $(2 \mathrm{M}$ GTC; $1 \%$ v/v SDS; $55 \%$ ethanol) was added to the column. The sample was again centrifuged for one minute at $1000 \times \mathrm{g}$ and the flow-through was discarded. Next, $700 \mu \mathrm{L}$ of low stringency wash buffer ( $80 \%$ ethanol) was added, followed by one minute of centrifuging at $1000 \times \mathrm{g}$. The flow-through was discarded, and the spin column was dried by centrifuging for one minute at $1000 \times \mathrm{g}$. Finally, $50 \mu \mathrm{L}$ of RNAase-free water was added to the column and was spun for thirty seconds at $1000 \times \mathrm{g}$. The resulting flow-through, containing the RNA, was then stored at $-80^{\circ} \mathrm{C}$.

\subsection{Cell Culture}

A549 human lung epithelial cells were obtained from the ATCC (CRM-CCL-185, 
Manassas, VA). The cells were cultured in RPMI-1640 media (Lonza, Basel, Switzerland) supplemented with $10 \%$ fetal bovine serum, $1 \%$ L-glutamine, and $1 \%$ penicillin-streptomycin and incubated at $37^{\circ} \mathrm{C}$ in $5 \% \mathrm{CO}_{2}$. To create a monolayer on the bottom of a six-well plate for the adherence and invasion assays, the cells were washed two times with phosphate-buffered saline (PBS) and detached from the flasks using $0.25 \%$ trypsin-EDTA (VWR, Radnor, PA). The suspended cells were seeded into a six-well plate at a density of $1 \times 10^{6}$ cells/well with $3 \mathrm{~mL}$ of the supplemented RPMI per well and were incubated for 24 hours at $37^{\circ} \mathrm{C}$ in $5 \% \mathrm{CO}_{2}$.

\subsection{Adhesion and Invasion Assay}

A culture of each serotype isolated at mid-logarithmic phase was added to $20 \mathrm{~mL}$ PBS supplemented with magnesium and calcium (PBS+/+), and $3 \mathrm{~mL}$ of these inocula were added to 6 wells for each serotype (multiplicity of infection $(\mathrm{MOI})=10)$. The plates were incubated for one hour at $37^{\circ} \mathrm{C}$ and $5 \% \mathrm{CO}_{2}$ and then the bacterial suspensions were removed, followed by two washes with PBS. Gentamicin Sulfate (Teknova, Hollister, CA) was added to three of the six wells in $100 \mu \mathrm{g} / \mathrm{mL}$ concentration. Because gentamicin is unable to diffuse across or be transported across the eukaryotic plasma membrane, it killed only extracellular bacteria, leaving those which had successfully entered the epithelial cells alive. Three wells per serotype examined the ability of the bacteria to invade the cells, while the remaining three wells examined adherence + invasion. After allowing the plates to incubate at room temperature for approximately ten minutes, the gentamicin was removed, and the plates were washed twice with PBS. Sterile water was added to all six wells to lyse the epithelial cells and release all intracellular bacteria. The bacteria were plated in triplicate on trypticase soy agar (TSA) with 5\% sheep blood (BD, Franklin Lakes, $\mathrm{NJ}$ ) and left to incubate overnight at $37^{\circ} \mathrm{C}$ and $5 \% \mathrm{CO}_{2}$. The number of colony-forming units (CFUs) was counted the following day.

\subsection{RNA Isolation from Cell Culture}

For cell culture RNA extraction, following the one hour incubation with the bacterial cultures as stated above, the cells were washed two times with PBS, but instead of the addition of gentamicin, $350 \mu \mathrm{L}$ of tri-reagent was added. Following the addition of the tri-reagent to the cell wells, the RNA was isolated from the cells as described above for the broth assay.

\subsection{Quantitative Reverse Transcriptase PCR (qRT-PCR)}

The mRNA obtained from the broth cultures and the cell cultures were quantified using the Qubit HS RNA kit and Qubit fluorometer (Invitrogen, Carlsbad, CA). $500 \mathrm{ng}$ of RNA was used as a template for synthesis of cDNA using the qScript ${ }^{\mathrm{TM}}$ cDNA SuperMix reagents and protocol (Quanta Biosciences, Beverly, MA). Real-time polymerase chain reaction (RT-PCR) reactions were set-up using the PerfeCTa qPCR reagent according to the manufacturer's protocol 
(Quanta Biosciences, Beverly, MA) using the primers listed in Table 1, and were cycled and analyzed using a Roche Light Cycler 480 (Basel, Switzerland). An initial 10 minute incubation at $50^{\circ} \mathrm{C}$ allowed for the conversion of the RNA to complementary DNA using reverse transcriptase, which was then followed by the following cycles for DNA amplification: 1 minute enature at $95^{\circ} \mathrm{C} ; 45$ cycles of 10 seconds at $95^{\circ} \mathrm{C}, 30$ seconds at $60^{\circ} \mathrm{C}$ with a quantification fluorescence read, and 30 seconds at $72^{\circ} \mathrm{C}$; followed by a melt curve of 10 seconds at $95^{\circ} \mathrm{C}, 30$ seconds at $40^{\circ} \mathrm{C}$ with continuous fluorescence readings to $95^{\circ} \mathrm{C}$; and a final cool down to $40^{\circ} \mathrm{C}$. qRT-PCR was used to determine the level of mRNA present for 6 genes: $c p s A, c b p A, p l y, l y t A$, and $s p b x$, and the $16 S r R N A$ gene, which served as an internal control. The data from the serotype $19 \mathrm{~F}$ was used for normalization purposes to establish a comparative fold change between serotypes using the delta-delta Ct methodsince this serotype was the slowest growing in culture, low in binding efficiency to the cultured lung cells, and had lowest gene expression in most assessments [15]. Melt analysis of the qRT-PCR amplicons was used to confirm PCR specificity.

\subsection{Statistical Analysis}

Graphing and statistical analysis was performed using GraphPad Prism 7 (version 7.02). To assess statistical significance between different serotypes a Kruskal-Wallis one-way ANOVA was used. In cases in which there was significant difference, a post-hoc analysis was performed using the Tukey Honestly Significant Difference method.

\section{Results}

\subsection{Comparison of Bacterial Growth}

Serotypes 5, $9 \mathrm{v}$, and $19 \mathrm{~F}$ behave similarly in trypticase soy broth, and gradually increase over an 8 hour period (Figure 1). Serotype 3, however, has an earlier and more abrupt exponential phase and enters the stationary phase by 5 hours (Figure 1). Serotype 3 reached the mid-logarithmic phase point used for RNA isolation $\left(\mathrm{OD}_{600}=0.250\right)$ by 3 hours, whereas the other 3 serotypes took a little over 5 hours to reach this point.

Table 1. Primer sequences for qRT-PCR. The primers were created by using PubMed database along with Primer3Plus software.

\begin{tabular}{ccc}
\hline Gene & Forward Primer & Reverse Primer \\
\hline$C p s A$ & ACGCAACTGACGAGTGTGAC & GATCGCGACACCGAACTAAT \\
$C b p A$ & CTTTCACATCGGACTCAGCA & CTTCCAAGCTCATCCCTGAA \\
$S p x B$ & ATTCGGCGGCTCAATCGGGG & CAGCACGGCAGGCTTCGTCA \\
$P l y$ & GCTTATGGGCGCCAAGTCTA & CAAAGCTTCAAAAGCAGCCTC \\
$L y t A$ & ACGCAATCTAGCAGATGAAGCA & TCGTGCGTTTTAATTCCAGCT \\
$16 S$ rRNA & CTGCGTTGTATTAGCTAGTTGGTG & TCCGTCCATTGCCGAAGATTC \\
\hline
\end{tabular}




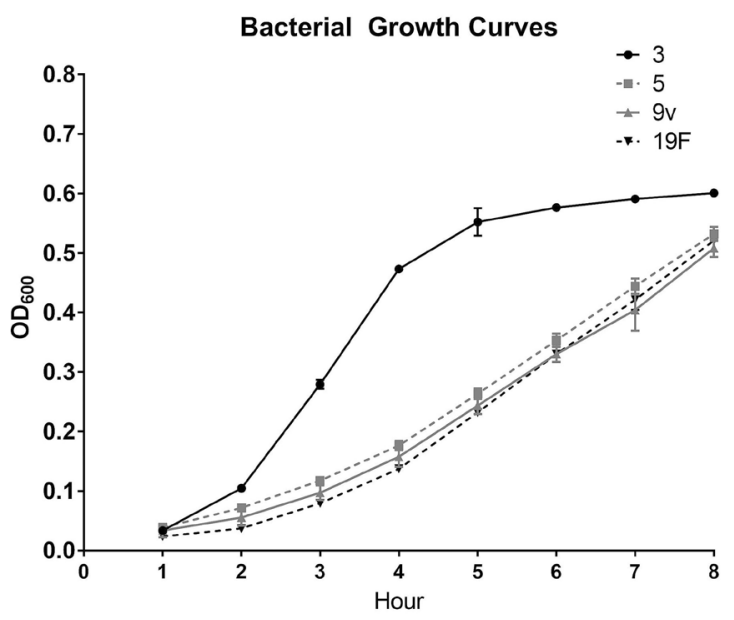

Figure 1. Bacterial growth curves in TSB. Ten milliliters $(\mathrm{mL})$ of trypticase soy broth (TSB) was inoculated with 200 microliters $(\mu \mathrm{L})$ of overnight culture $(n=4)$ for each serotype. The bacteria were grown at $37^{\circ} \mathrm{C}$ and every hour for 8 hours $1 \mathrm{~mL}$ of culture was read by spectrophotometry to determine the absorbance at an optical density of $600 \mathrm{~nm}$.

\subsection{Comparison of Gene Expression in Broth Culture}

$19 \mathrm{~F}$ was selected as a base-line for the gene expression data since the replicates were very close in this serotype, and it had lower gene expression for nearly every gene tested. The $9 \mathrm{v}$ expression of the autolysin gene (lyt $A$ ) was very close in expression to the $19 \mathrm{~F}$, but an increase in expression was observed with the serotypes 3 and 5, with a 5.5 and 7.8-fold increase, respectively (Figure 2(a)). Only the serotype 5 increase was significant due to the variation observed in the serotype 3 expression (Figure 2(a)). An increase in pneumolysin gene ( $p l y)$ gene expression was observed in all of the serotypes over $19 \mathrm{~F}$, with a 9-fold, 3.6-fold, and 2.6-fold increase in serotypes 3,5, and $9 \mathrm{v}$, respectively (Figure 2(b)), but only the serotype 3 increase was statistically significant. Choline binding protein gene $(c b p A)$ expression was very similar in levels between most samples, but a few outliers in the $9 \mathrm{v}$ serotype did result in a significant difference in expression between this serotype and serotype 3 (Figure $2(c)$ ). The genes for hydrogen peroxide production $(s p x B)$ and capsule production $(\operatorname{cps} A)$ had very little variation between samples (Figure 2(d) and Figure 2(e)).

\subsection{Adherence and Invasion of A549 Cells}

Gentamicin sulfate does not cross eukaryotic cell membranes, and thus only kills extracellular bacteria. The addition of gentamicin allows for the determination of the ability of pneumococcal cells to invade A549 lung epithelial cells. Without gentamicin, the cell counts will include both adherent and internalized cells. Approximately twice as much bacteria were present in the gentamicin negative culture for serotype $9 \mathrm{v}$, suggesting that about half $(\sim 46 \%)$ of the bacteria successfully invaded the epithelial cells (Figure 3). $19 \mathrm{~F}$ also had a little over half of the culture residing inside of the cells (58\%) (Figure 3). Although $9 \mathrm{v}$ colonized 


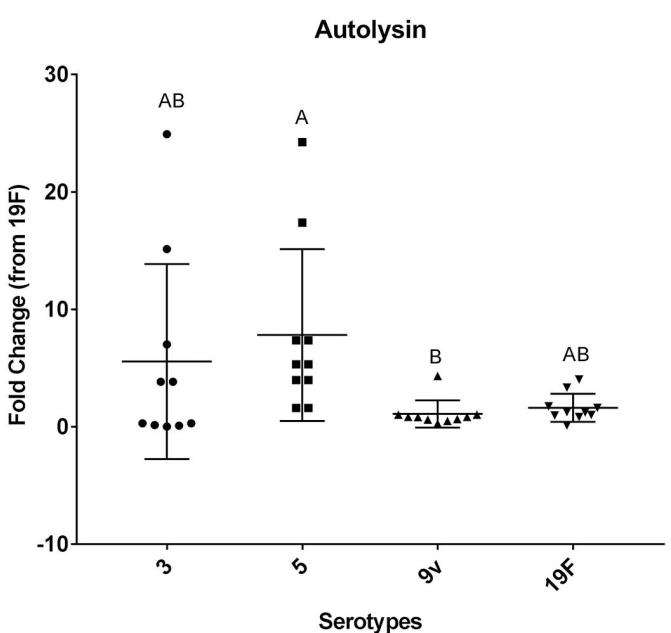

(a)

Choline Binding Protein

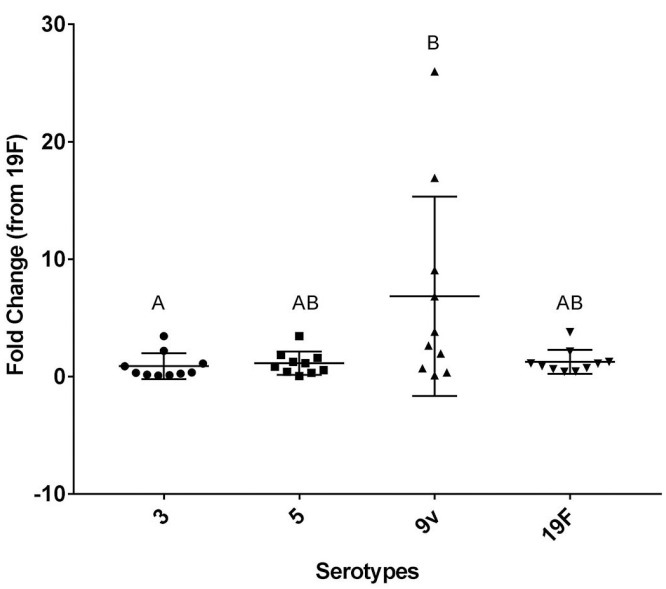

(c)

Capsule (A)

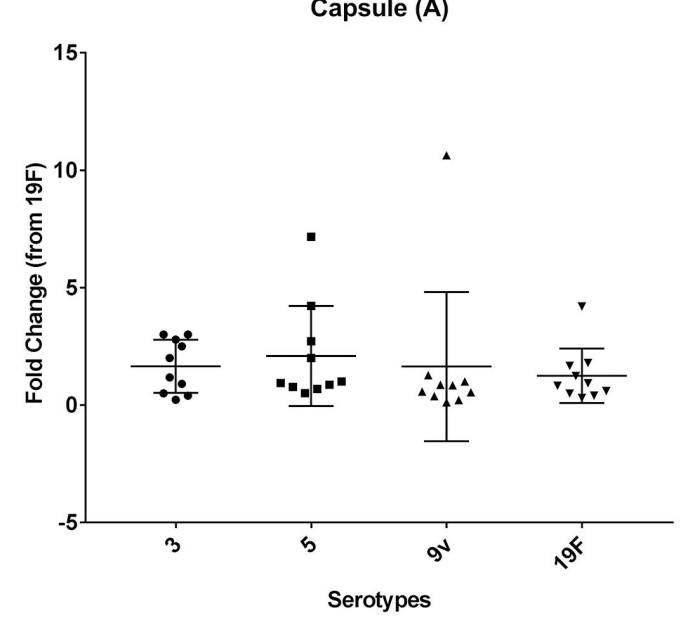

(e)

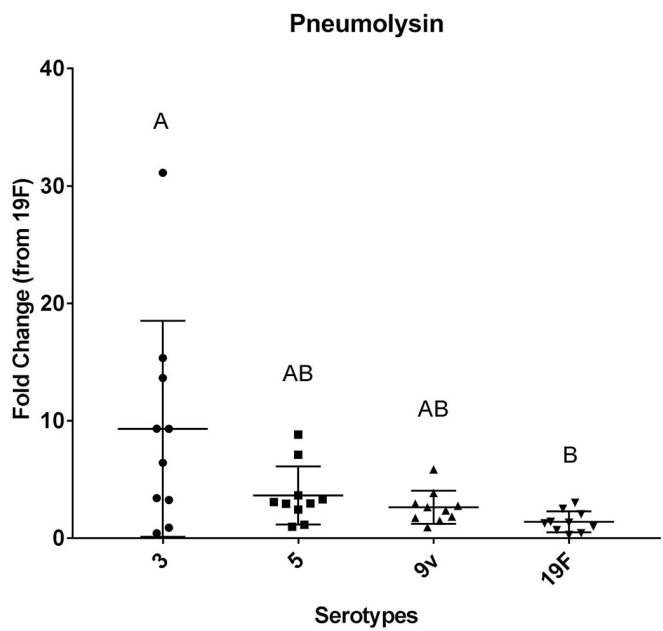

(b)

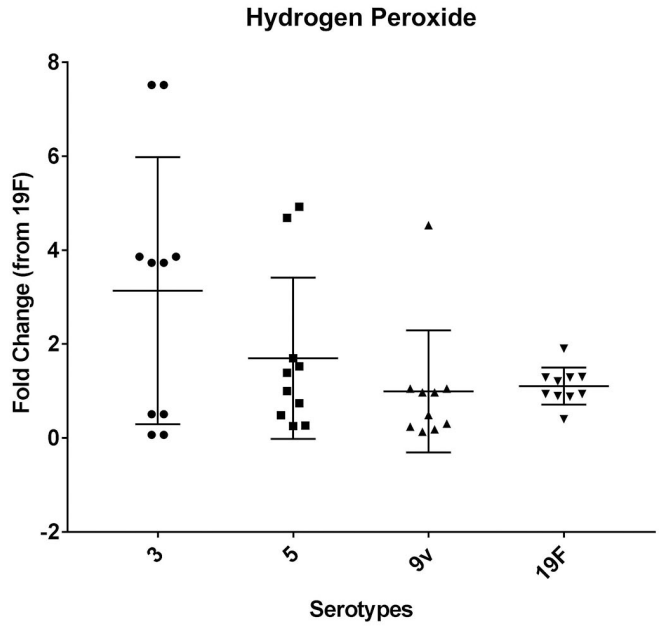

(d)

Figure 2. Bacterial gene expression following growth in TSB. The four serotypes of $S$. pneumoniae were grown to mid-logarithmic phase in TSB, and following RNA isolation, gene expression was assessed and compared for the autolysin (a), pneumolysin (b), choline binding protein (c), hydrogen peroxide production (d), and the first gene of the capsule operon $(\mathrm{E})(\mathrm{n}=10)$. Statistical differences are denoted with differing letters when $\mathrm{p}<0.05$ as determined via ANOVA. 


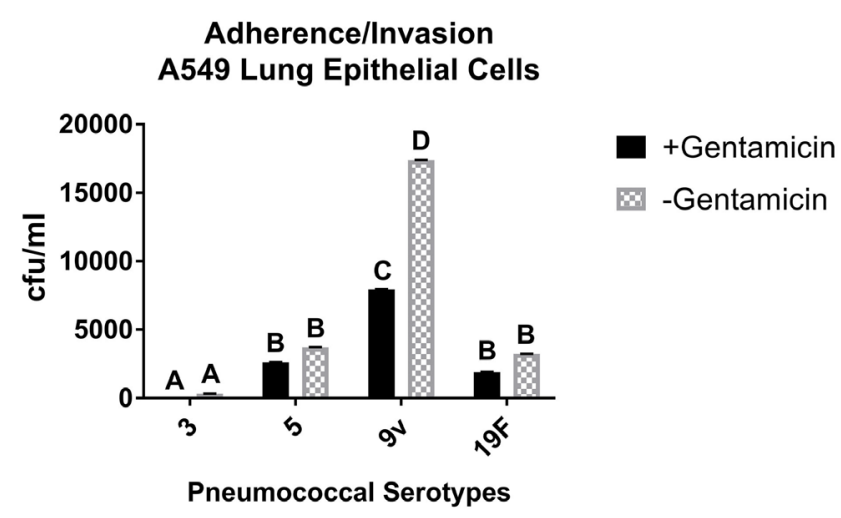

Figure 3. Pneumococcal adherence and invasion in lung epithelial cells. The four $S$. pneumoniae serotypes were grown to mid-logarithmic phase and added to A549 lung epithelial cells at an MOI of 10. Following a 1 hour incubation, gentamicin was added to half of the wells $(n=4)$ to kill extracellular bacteria. The wells with and without gentamicin addition were compared among the four serotypes. Different letters indicate a significant change with $\mathrm{p}<0.05$ as determined via ANOVA.

the epithelial cells slightly better, serotype 5 showed a slightly better ability to invade ( $70 \%$ ) (Figure 3 ). Serotype 3 colonized the lung cells very poorly and was not capable of cell invasion (Figure 3 ).

\subsection{Gene Expression in S. pneumoniae-Infected A549 Cells}

Although a little more variation was observed in the expression by serotype 3 , there was no significant change in expression of the autolysin between the four serotypes (Figure 4(a)). Expression of the pneumolysin was significantly increased in the serotypes 3 and 5 over $19 \mathrm{~F}$ when cultured with A549 cells, but expression was not significantly higher than the serotype $9 \mathrm{v}$ (Figure 4(b)). Serotypes 3 and 5 also showed similar expression of the choline binding protein $\mathrm{A}$, and both had significantly greater expression than $9 \mathrm{v}$ and $19 \mathrm{~F}$ (Figure 4(c)). There was no significant change in hydrogen peroxide gene expression (Figure 4(d)), and the capsule expression was only significantly upregulated in the serotype 5 bacteria when exposed to A549 cells (Figure 4(e)).

\subsection{Comparison of Gene Expression between Broth and Cells}

When using the broth critical point values for determining the fold change of cell culture gene expression, it was concluded for the most part the pneumococci gene expression in cell culture is lower than what it is in broth culture (Figure 5). Expression of the autolysin, pneumolysin, and choline binding protein $A$ genes were all downregulated in cell culture when compared to broth culture by all four serotypes of Streptococcus pneumoniae used in this study (Figures 5(a)-(c)). The hydrogen peroxide gene expression was downregulated in cell culture for all serotypes except for 5, which had a lot of variation within the cell culture data (Figure 5(d)), and expression of the first gene of the capsule operon was downregulated in serotypes 5 and $19 \mathrm{~F}$ (Figure 5(e)). 


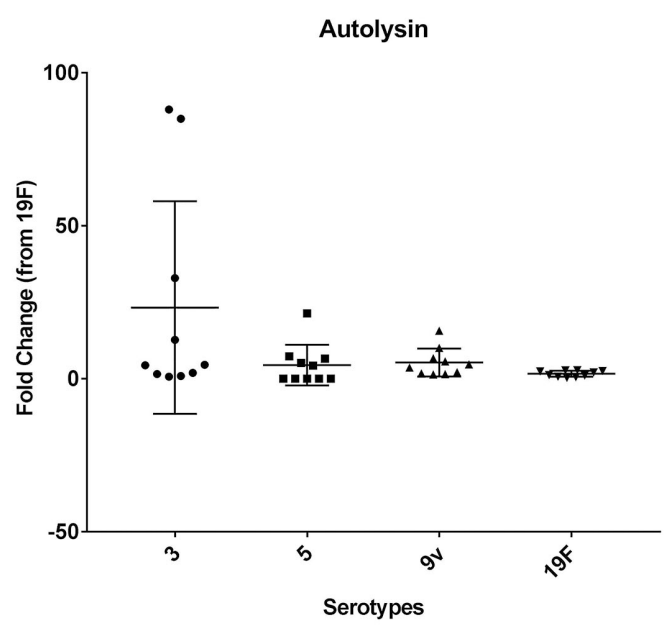

(a)

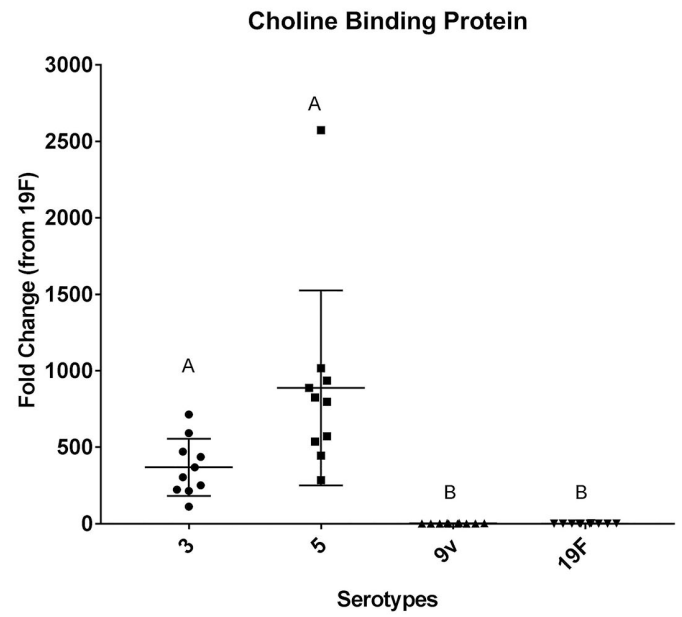

(c)

Capsule (A)

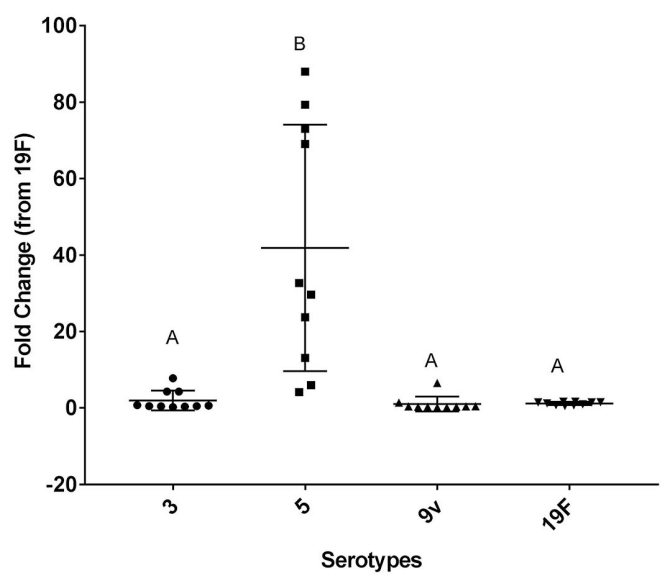

(e)

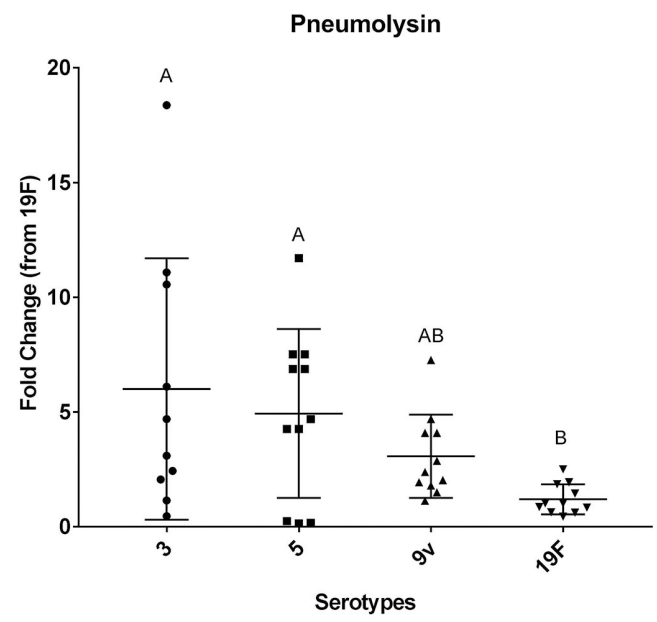

(b)

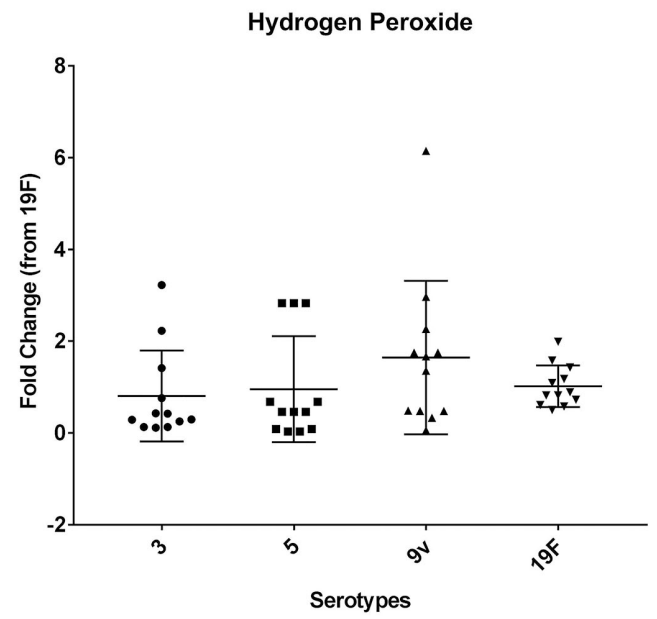

(d)

Figure 4. Bacterial gene expression following 1 hour exposure to lung epithelial cells. The four serotypes of $S$. pneumoniae were grown to mid-logarithmic phase in TSB, and added to A549 cells for 1 hour at an MOI of 10. Following incubation, RNA was isolated and gene expression was assessed and compared for the autolysin (a), pneumolysin (b), choline binding protein (c), hydrogen peroxide production (d), and the first gene of the capsule operon $(\mathrm{e})(\mathrm{n}=10)$. Statistical differences are denoted with differing letters when $\mathrm{p}<0.05$ as determined via ANOVA. 


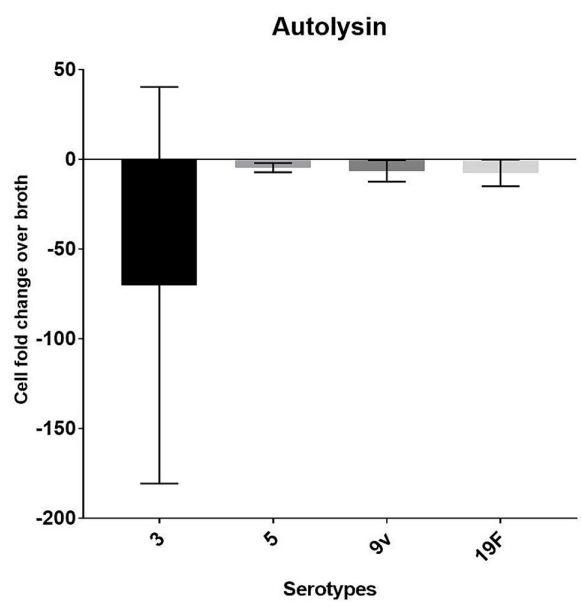

(a)

Choline Binding Protein A

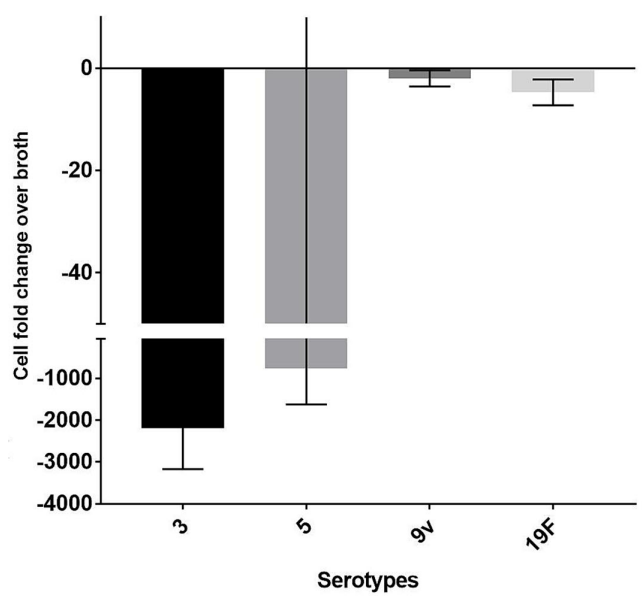

(c)

Capsule (A)

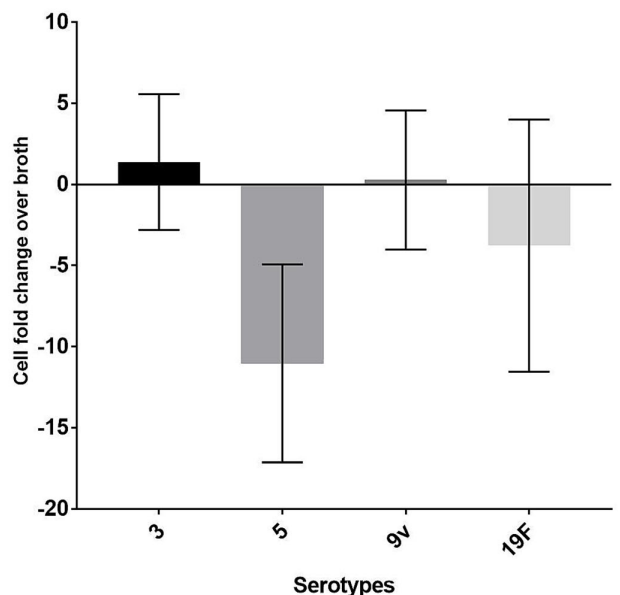

(e)

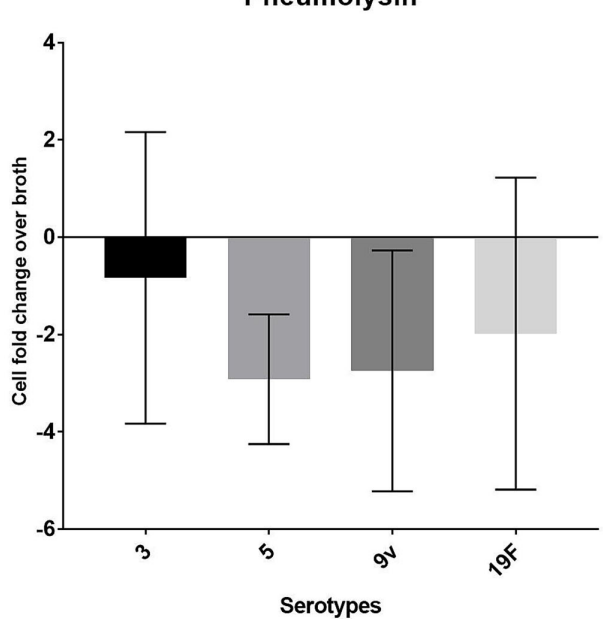

(b)

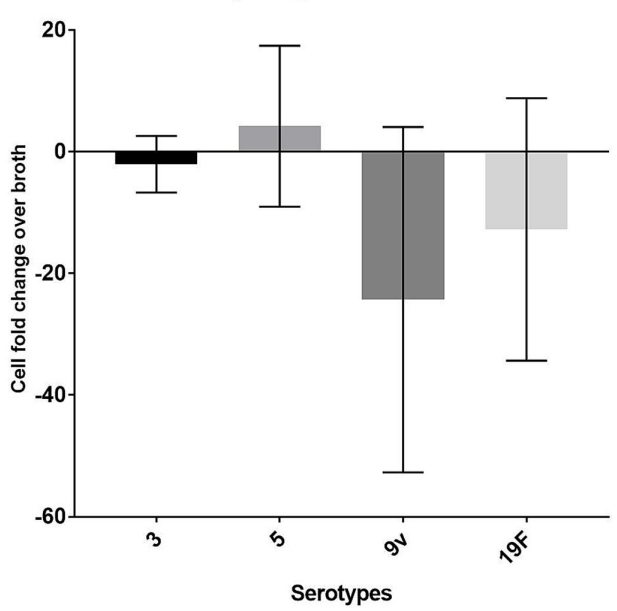

(d)

Figure 5. Gene expression comparison of cell culture versus broth culture. Using the delta-delta Ct method and the broth critical threshold (Ct) values as a control, the data was re-analyzed to show a comparison of gene expression between the normalized $\mathrm{Ct}$ values obtained from $S$. pneumoniae isolates exposed to cell cultures versus those only grown in broth culture. 


\section{Discussion}

Serotypes 5, $9 \mathrm{v}$, and $19 \mathrm{~F}$ grew at a similar rate in broth culture (Figure 1), and with the exception of an upregulated autolysin expression in serotype 5, their gene expression was very similar too (Figure 2). Serotype 3 had a more rapid entrance into exponential phase (Figure 1), and demonstrated slightly upregulated autolysin and pneumolysin expression compared to the other serotypes (Figure 2), but for the most part, any of these serotypes could serve as a representative serotype for pneumococcal gene expression in broth culture.

In the lung epithelial cultures using A549 cells, again serotypes 5, $9 \mathrm{v}$, and $19 \mathrm{~F}$ behaved quite similarly in both adherence and invasion of A549 cells (Figure 3), while serotype 3 did not adhere as well or invade at all (Figure 3 ). Since serotype 3 produced far more choline binding protein than serotypes $9 \mathrm{v}$ and $19 \mathrm{~F}$ (Figure $4(c)$ ), it was surprising to see that it did not colonize as well to lung epithelial cells since A549 cells have choline in their phospholipid bilayer [16], especially since it is known to cause invasive infections in humans [17]. Therefore it is hypothesized that the capsule may have impacted the ability of these four serotypes to colonize lung epithelial cells, and not the binding proteins. Although three of the four serotypes produced similar levels of $\operatorname{cps} A$ mRNA, with only serotype 5 being upregulated in the lung cell culture (Figure 4(e)), the composition of the capsule likely impacted colonization and invasion potential, and the regulation of the capsule may be different in vivo than in transformed cell lines.

There are 90 characterized serotypes of pneumococcal capsule, and several ungroupable structures, most of which are anionic, and thus repel from cells [18]. The serotypes vary in their ability to cause disease. Serotype 3 is frequently associated with invasive pneumococcal disease (IPD) in the elderly, and has a high risk of mortality [18]. It however has been shown to have a poor carriage rate in humans [19]. The metabolic cost of producing the very thick capsule associated with serotype 3 pneumococci has been suggested to impact its presence in the normal microflora [19]. Serotype 5 has a low carriage rate, but has a high association with IPD, although not as fatal [19]. Serotype $9 \mathrm{v}$ has a high carriage, but low mortality rate, while $19 \mathrm{~F}$ has a high carriage and high mortality rate, especially in children [19].

There was a lot of variation in the autolysin and pneumolysin expression from the serotype 3 pneumococci when exposed to A549 cells, which could reflect those cell cultures that had higher colonization rates (Figure 4(a) and Figure 4(b)). Serotype 3 had higher average expression of these two virulence factors, although only the pneumolysin was significant due to high levels of variation (Figure 4(a) and Figure 4(b)). Serotype 5 had significantly increased expression of pneumolysin over $19 \mathrm{~F}$, while $9 \mathrm{v}$ had slightly elevated expression (Figure 4 (b)), which is surprising since $9 \mathrm{v}$ has a lower mortality rate than $19 \mathrm{~F}$ [20]. The expression levels for the gene that regulates hydrogen peroxide production had no variation among the strains in cells or broth (Figure 2(d) and Figure 4(d)) suggesting that this virulence factor is produced by a variety of strains, or is only 
essential in vivo.

Since the pathobiology of $S$. pneumoniae differs greatly between various serotypes, there have been many attempts to better understand differential gene regulation between the various serogroups [21] [22] [23]. However, a majority of these attempts utilize bacteria grown in broth culture, and typically only one serotype [21] [22] [23]. A lot of the research performed still relies on Avery's D39 serotype 2 strain [22] [23] because of its ease of transformation and virulence in mice [24]. However, serotype 2 is rare in humans and not associated with IPD clinical cases [25] [26]. It is important to understand how other strains interact with human cells to cause colonization and infection, especially for the more common and more pathogenic serotypes. We have demonstrated here that the serotypes can differ in their ability to regulate various genes (Figure 2 and Figure 4), and that host cells can greatly influence the regulation of gene expression by $S$. pneumoniae (Figure 5). We examined frequent colonizers of humans (5 and $19 \mathrm{~F}$ ) with two that have been highly implicated in adult IPD cases ( 3 and $9 \mathrm{v}$ ), although the epidemiological data for $S$. pneumoniae infection does vary dramatically depending on the source country [24] [25] [26], and invasive strains have been isolated for all four serogroups [26], suggesting variation within the serogroups. For being more frequently implicated in invasive diseases, serotype $9 \mathrm{v}$ behaved similarly to $19 \mathrm{~F}$ in both broth and cell culture not only in gene expression, but also in growth and adhesion and invasion abilities (Figures 1-4). Serotype 5 grew similarly in broth and had similar gene expression to $9 \mathrm{v}$ and $19 \mathrm{~F}$ when grown in broth (Figures 1-2), but differed more when grown in cell culture. It adhered and invaded at a similar level (Figure 3), but serotype 5 had a lot of variability in gene expression when exposed to A549 cells, and had significant upregulations of choline binding proteins and capsule expression compared with $9 \mathrm{v}$ and $19 \mathrm{~F}$ (Figure 4), suggesting that for this particular serotype, these virulence factors may be important for cell colonization. Serotype 3 differed greatly in its ability to grow in broth culture (Figure 1 and Figure 3), and had increased pneumolysin and autolysin activity (Figure 2 and Figure 4) compared to the other serotypes, which likely contributes to its frequency of IPD.

Although there are many more serotypes and virulence factors that can be studied with the pneumococcus, it is demonstrated here that studying gene regulation with this bacterial species can be challenging due to the diversity and variability of the species. Various strains behave differently from one another in the same medium, and can vary dramatically when observed in different culture conditions. Host cell factors impact the way that the pneumococci regulate their genes, and surprisingly most bacterial genes that we examined here were down-regulated in the presence of the host cells as compared to growth in broth (Figure 5). It was hypothesized that we would see the exact opposite effect since all the genes that were included in this assay are known virulence factors for $S$. pneumoniae, and thus we thought that the presence of host cells would increase the expression, and not decrease it. This again reiterates the need to study bacte- 
ria in a more natural system since understanding gene regulation is most important when the bacteria are in their natural setting.

\section{Acknowledgements}

We would like to thank the Offices of Student Research and Creative Activities and Faculty Research of Tarleton State University for funding this project. All biosafety protocols were approved by the Institutional Biosafety Committee at Tarleton State University, and were strictly adhered to for the pneumococci and human cell line.

\section{Conflicts of Interest}

The authors declare no conflicts of interest regarding the publication of this paper.

\section{References}

[1] Frankel, R., Virata, M., Hardalo, C., Altice, F. and Friedland, G. (1996) Invasive Pneumococcal Disease: Clinical Features, Serotypes, and Antimicrobial Resistance Patterns in Cases Involving Patients with and without Human Immunodeficiency Virus Infection. Clinical Infectious Diseases, 23, 577-584. https://doi.org/10.1093/clinids/23.3.577

[2] Alanee, S., McGee, L., Jackson, D., Chiou, C., Feldman, C., et al. (2007) Association of Serotypes of Streptococcus pneumoniae with Disease Severity and Outcome in Adults: An International Study. Clinical Infectious Diseases, 45, 46-51. https://doi.org/10.1086/518538

[3] Hamborsky, J., Kroger, A. and Wolfe, S. (2015) Epidemiology and Prevention of Vaccine-Preventable Diseases. 13rd Edition, Centers for Disease Control and Prevention, Public Health Foundation, Washington DC.

[4] Shiri, T., Datta, S., Madan, J., Tsertsvadze, A., Royle, P., et al. (2017) Indirect Effects of Childhood Pneumococcal Conjugate Vaccination in Invasive Pneumococcal Disease: A Systematic Review and Meta-Analysis. The Lancet, 5, e51-e59. https://doi.org/10.1016/S2214-109X(16)30306-0

[5] O’Brien, K., Wolfson, L., Watt, J., Henkle, E., Deloria-Knoll, M., et al. (2009) Burden of Disease Caused by Streptococcus pneumoniae in Children Younger than 5 Years: Global Estimates. The Lancet, 374, 893-902. https://doi.org/10.1016/S0140-6736(09)61204-6

[6] Henrichsen, J. (1995) Six Newly Recognized Types of Streptococcus pneumoniae. Journal of Clinical Microbiology, 33, 2759-2762.

[7] Sjöström, K., Spindler, C., Ortqvist, A., Kalin, M., Sandgren, A., et al. (2006) Clonal and Capsular Types Decide Whether Pneumococci Will Act as a Primary or Opportunistic Pathogen. Clinical Infectious Diseases, 42, 451-459. https://doi.org/10.1086/499242

[8] Morona, J., Morona, R. and Paton, J. (2006) Attachment of Capsular Polysaccharide to the Cell Wall of Streptococcus pneumoniae Type 2 Is Required for Invasive Disease. PNAS, 103, 8505-8510. https://doi.org/10.1073/pnas.0602148103

[9] Rensheng, L., Mann, B., Lewis, W., Rowe, A., Heath, R., et al. (2005) Solution Structure of Choline Binding Protein A, the Major Adhesin of Streptococcus pneumoniae. The EMBO Journal, 24, 34-43. 
https://doi.org/10.1038/sj.emboj.7600490

[10] Howard, L. and Gooder, H. (1974) Specificity of the Autolysin of Streptococcus (Diplococcus) pneumoniae. Journal of Bacteriology, 117, 796-804.

[11] Hirst, R., Kadioglu, A., O'Callaghan, C. and Andrew, P. (2004) The Role of Pneumolysin in Pneumococcal Pneumonia and Meningitis. Clinical \& Experimental Immunology, 138, 195-201. https://doi.org/10.1111/j.1365-2249.2004.02611.X

[12] García-Suárez, M., Flórez, N., Astudillo, A., Vázquez, F., Villaverde, R., Fabrizio, K., et al. (2007) The Role of Pnuemolysin in Mediating Lung Damage in a Lethal Pneumococcal Pneumonia Murine Model. Respiratory Research, 8, 3.

[13] Echlin, H., Frank, M., Iverson, A., Chang, T., Johnson, M., et al. (2016) Pyruvate Oxidase as a Critical Link between Metabolism and Capsule Biosynthesis in Streptococcus pneumoniae. PLoS Pathology, 12, e1005951. https://doi.org/10.1371/journal.ppat.1005951

[14] Carvalho, S., Andisi, V., Gradstedt, H., Neef, J., Kuipers, O., et al. (2013) Pyruvate Oxidase Influences the Sugar Utilization Pattern and Capsule Production in Streptococcus pneumoniae. PLoS ONE, 8, e68277. https://doi.org/10.1371/journal.pone.0068277

[15] Livak, K.J. and Schmittgen, T.D. (2001) Analysis of Relative Gene Expression Data Using Real-Time Quantitative PCR and the 2(-Delta Delta C(T)) Method. Methods, 25, 402-408.

[16] Matsumura, Y., Sakai, H., Ban, N. and Inagaki, N. (2007) ABECA3-Mediated Choline Phospholipids Uptake into Intracellular Vesicles in A549 Cells. FEBS Letters, 581, 3139-3144. https://doi.org/10.1016/j.febslet.2007.05.078

[17] Binsker, U., Kohler, T.P., Krauel, K., Kohler, S., Habermeyer, J., et al. (2017) Serotype 3 Pneumococci Sequester Platelet-Derived Human Thrombospondin-1 via the Adhesin and Immune Evasion Protein Hic. The Journal of Biological Chemistry, 292, 5770-5783. https://doi.org/10.1074/jbc.M116.760504

[18] Gen, K.A., Gilbert, G.L., Song, J.Y., Skovsted, I.C., Klungman, K.P., et al. (2015) Pneumococcal Capsules and Their Types: Past, Present, and Future. Clinical Microbiology Reviews, 28, 871-899. https://doi.org/10.1128/CMR.00024-15

[19] Weingerger, D.M., Trzcinski, K., Lu, Y.-J., Bogaert, D., Brandes, A., et al. (2009) Pneumococcal Capsular Polysaccharide Structure Predicts Serotype Prevalence. PLoS Pathogens, 5, e1000479.

[20] Peterson, S., Cline, R.T., Tettelin, H., Sharov, V. and Morrison, D.A. (2000) Gene Expression Analysis of the Streptococcus pneumoniae Competence Regulons by Use of DNA Microarrays. Journal of Bacteriology, 182, 6192-6202. https://doi.org/10.1128/JB.182.21.6192-6202.2000

[21] Hoover, S.E., Perez, A.J., Tsui, H.-C., Sinha, D., Smiley, D.L., et al. (2015) A New Quorum-Sensing System (TprA/PhrA) for Streptococcus pneumoniae D39 That Regulates a Lantibiotic Biosynthesis Gene Cluster. Molecular Microbiology, 97, 229-243. https://doi.org/10.1111/mmi.13029

[22] Sorg, R.A., Kuipers, O.P. and Veening, J.W. (2014) Gene Expression Platform for Synthetic Biology in the Human Pathogen Streptococcus pneumoniae. ACS Synthetic Biology, 4, 228-239. https://doi.org/10.1021/sb500229s

[23] Lanie, J.A., Ng, W.L., Kazmierczak, K.M., Andrzejewski, T.M., Davidsen, T.M., et al. (2007) Genome Sequence of Avery's Virulent Serotype 2 Strain D39 of Streptococcus pneumoniae and Comparison with That of Unencapsulated Laboratory Strain R6. Journal of Bacteriology, 189, 38-51. https://doi.org/10.1128/JB.01148-06 
[24] Kalin, M. (1998) Pneumococcal Serotypes and Their Clinical Relevance. Thorax, 53, 159-162. https://doi.org/10.1136/thx.53.3.159

[25] Song, J.Y., Nahm, M.H. and Moseley, M.A. (2013) Clinical Implications of Pneumococcal Serotypes: Invasive Disease Potential, Clinical Presentations, and Antibiotic Resistance. Journal of Korean Medical Science, 28, 4-15.

https://doi.org/10.3346/jkms.2013.28.1.4

[26] Hausdorff, W.P., Bryant, J., Paradiso, P.R. and Siber, G.R. (2000) Which Pneumococcal Serotypes Cause the Most Invasive Disease: Implications for Conjugate Vaccine Formulation and Use? Clinical Infectious Diseases, 30, 100-121.

https://doi.org/10.1086/313608 